Innovación y Desarrollo Tecnológico y Social (2022) 4: 1-19

\title{
Integración de antropología, salud y tecnologías informáticas en una innovación tecnológico-social para el acompañamiento de personas con Enfermedad de Parkinson
}

Bacigalupe, María de los Angeles 1,2,3; Peñalva, Mirta ${ }^{1}$; Pujol, Silvana ${ }^{1}$

${ }^{1}$ MAB: CONICET, División Etnografía, Museo de La Plata. FCNyM, UNLP, MP: GIDAS, UTN-RLP, SP: Facultad de Ciencias Médicas, UNLP; ${ }^{2}$ Facultad de Ciencias Médicas, UNLP; ${ }^{3}$ mariabacigalupe@conicet.gov.ar

Resumen. Presentamos la estrategia de integración interdisciplinar para el acompañamiento de personas con Enfermedad de Parkinson (EP) que tiene como punto de partida un grupo de soporte social y físico denominado Taller de Parkinson (TdP) que se desarrolla como programa universitario de extensión. Las particularidades del desarrollo como innovación tecnológica y social tienen que ver con: 1. La metodología interdisciplinaria, 2. El marco teórico paradojal como herramienta terapéutica, 3. La apropiación del grupo originalmente destinatario (en vez de ser para es de), 4. El origen comunitario y no académico de la iniciativa y 5 . La incorporación de las tecnologías informáticas como contribución para el desarrollo de una aplicación de seguimiento de la salud de los participantes. Como cuestión no menor, a la superación de fronteras disciplinares y apropiación del grupo se sumó la no menos importante declaración de pandemia por COVID-19 con las consecuencias en el cierre de actividades presenciales y adaptaciones a la virtualidad.

Palabras clave: integración interdisciplinaria; enfoque antropológico-relacional; grupo de soporte físico y social; kinesia paradojal; Enfermedad de Parkinson

Recibido: 31/10/2021 Aceptado: 30/12/2021 - DOI: https://doi.org/10.24215/26838559e031 
Innovación y Desarrollo Tecnológico y Social (2022) 4: 1-19

Integration of anthropology, health, and computational technologies into a social and technological innovation to work with people with Parkinson's disease

Abstract. In this article we show the interdisciplinary strategy to work with people with Parkinson's disease (PD) which has its start point in the social and physical support group called Parkinson's Workshop (PW) -a university communitarian program. As a social and technological innovation, this program has some key features: 1. The logic of the interdisciplinarity as methodology, 2 . The framework of paradoxical kinesia as a therapeutic tool, 3. The empowerment of people with PD and their feeling of being part of the project (instead of being just receptors), 4. The community as a source of the project (instead the academia), and 5. The addition of the computing technologies to develop a software to monitor people with PD' health and generate new information. It is noteworthy to say that the COVID-19 pandemic emerged as a new and never before thought challenge that added a sui generis condition to the characteristics enumerated before, which made us develop new tools and virtual ways to communicate with people and continue activities.

Keywords: interdisciplinary integration; anthropological-relational approach; physical and social support group; paradoxical kinesia; Parkinson's disease

\section{Novedad u originalidad local en el conocimiento}

La puesta en marcha del grupo de soporte físico y social Taller de Parkinson (TdP) en 2002 constituyó un emprendimiento totalmente innovador con un marco teórico y metodológico original. Localmente no existía nada similar y las iniciativas que iban surgiendo tenían que ver más con grupos de autoayuda que con propuestas de actividad física especialmente diseñadas para personas con Enfermedad de Parkinson (EP). La kinesia paradojal se conocía asociada a lo fenomenológico de la enfermedad y como algo más anecdótico, sí se habían propuesto algunos mecanismos fisiológicos posibles (cfr. por ejemplo Keefe et al, 1989) pero, al menos dentro de nuestro conocimiento según las búsquedas realizadas, no existía antecedente que la propusiera como herramienta terapéutica. El único antecedente 
más remoto que conocemos de indagación de las propiedades del movimiento paradojal como instrumento de acción es el del Prof. David Lee trabajando con Ivan Vaughan (Prof. S. Valenti [Hofstra University], comunicación personal, 8/7/20, pueden verse dos videos sobre este trabajo en Perception Movement Action Research Consortium, s/d).

No fue sino en la segunda década de este siglo que comenzaron a aparecer algunas propuestas multidisciplinarias locales dirigidas a las personas con Parkinson. Sin embargo, a la fecha no se conoce otra propuesta local con el marco teórico y metodológico de la innovación que presentamos, aunque sí hay mayor oferta de rehabilitación por el ejercicio, la fonoaudiología y la kinesiología para personas con EP.

\section{Grado de relevancia}

La EP constituye el trastorno del movimiento más común y ocupa el segundo lugar entre los trastornos neurodegenerativos; afecta al 1 o 2 por mil de la población y su prevalencia se incrementa a medida que aumenta la edad del grupo etario considerado, afectando el 1\% de la población por encima de 60 años (Tysnes \& Storstein, 2017).

No contamos con datos epidemiológicos actuales de prevalencia de la EP en la región, aunque podemos mencionar un estudio latinoamericano publicado recientemente (Vial et al, 2021) donde se encontró que en Chile en 2018 la prevalencia cruda era de 160,7 por cada cien mil personas y que la razón varonesmujeres era de 1,03. En un estudio realizado en Buenos Aires, Argentina, se halló que la prevalencia a diciembre de 2008 era de 219 cada cien mil habitantes en la población general y de 394 cada cien mil habitantes en la población mayor de 40 años (Bauso et al, 2012).

Estos datos dan cuenta de la relevancia del problema dentro de la salud poblacional, algo reconocido a nivel de políticas públicas de salud (cfr. por ejemplo Disposición 
Innovación y Desarrollo Tecnológico y Social (2022) 4: 1-19

1838/2007 del Servicio Nacional de Rehabilitación y Resolución 753/2007 del Ministerio de Salud) aunque con un desarrollo bastante escaso con referencia a lo que ocurre en otros países.

Consideramos que el objetivo del TdP, mejorar la calidad de vida de las personas con EP, se inserta en un campo problemático amplio y con potencialidad de desarrollo y cambio. A nivel local existen otros programas de calidad de vida que, con características distintas, se inscriben en el mismo marco problemático, como el Programa de Parkinson y Movimientos Anormales desarrollado en el Hospital de Clínicas de Buenos Aires (http://neurologia.hospitaldeclinicas.uba.ar/ y https://www.facebook.com/programadeparkinson/).

\section{Grado de pertinencia}

La innovación surgió desde el mismo seno de la comunidad y la institución de salud. Su crecimiento se asoció a las posibilidades de la Universidad de brindar un marco institucional apropiado donde los saberes de los practicantes de la salud se unieron a las posibilidades de la extensión universitaria. Finalmente, para responder con mayor pertinencia a algunos rasgos que surgieron posteriormente, fue que se introdujo a las tecnologías de la información y la comunicación y a especialistas en ciencias de la computación. De este modo, la pertinencia de la innovación tecnológico-social que presentamos para el abordaje de la problemática de salud de las personas con EP es adecuada, trabajando conjuntamente desde el área de extensión e investigación y desarrollo e incorporando a actores de la comunidad como practicantes de la institución sanitaria. Esta pertinencia es relativa en tanto son necesarios esfuerzos conjuntos de distintos actores institucionales y comunitarios para mejorar la calidad de vida de las personas con Parkinson. La innovación que representa el TdP es uno de esos actores que, consideramos, aporta una mirada original y necesaria en el abordaje de la problemática. 
Innovación y Desarrollo Tecnológico y Social (2022) 4: 1-19

\section{Grado de demanda}

El grupo de soporte TdP surgió a partir de la iniciativa de quien era jefe del Servicio de Neurología del Hospital Dr. A. Korn (Melchor Romero, Provincia de Buenos Aires, Argentina), José Luis Dillon, como respuesta a una demanda que observaba en los pacientes ambulatorios con Parkinson del Servicio. De este modo, la demanda surgió de la propia comunidad de personas afectadas con la enfermedad y la innovación tecnológico-social que presentamos vino a responder a esa demanda. Conociendo la alta prevalencia de la EP en la población, su condición de incurable y crónica y la respuesta que a nivel local hemos tenido tanto del grupo de personas con EP y familias como de la UNLP apoyando la iniciativa, consideramos que abunda la demanda y que este emprendimiento presenta una alta potencialidad para atender esta demanda existente.

Podemos dar cuenta del interés poblacional a partir de la respuesta de los medios de comunicación masiva; en una subsección de la sección final de referencias citamos algunas entrevistas realizadas en estos medios.

\section{Desarrollo del producto}

\section{Introducción y objetivo}

La Enfermedad de Parkinson (EP) constituye una condición de salud multisistémica que incluye no sólo la afección de distintos sistemas biológicos sino la comunicación con el entorno de las personas afectadas. Su prevalencia es alta y aumenta a medida que aumenta el grupo etario que se considera. Como afección neurológica, se clasifica dentro de los trastornos neurodegenerativos del movimiento.

En el año 2002 surgió el espacio denominado Taller de Parkinson (TdP) como propuesta a la demanda de personas con Enfermedad de Parkinson de algo más que el tratamiento neurológico que pudiera hacerse para mejorar su salud. Un grupo de profesionales liderado por el neurólogo José Luis Dillon fue quien gestionó el proyecto desde una institución pública de salud. La propuesta se constituyó como 
Innovación y Desarrollo Tecnológico y Social (2022) 4: 1-19

una innovación tendiente al soporte físico y social complementario al tratamiento neurológico de la persona con Parkinson.

A partir de 2006 la propuesta se sumó a las convocatorias de extensión universitaria de la Universidad Nacional de La Plata (UNLP) y actualmente constituye un programa estable de extensión de la Facultad de Ciencias Médicas (FCM) y un proyecto de la UNLP.

El programa está fundamentado en dos pilares teórico-metodológicos: el fenómeno paradojal y el abordaje interdisciplinario.

Si bien actualmente el uso de distintas terapias complementarias para las personas con EP está bastante difundido, en el momento de creación del TdP resultó totalmente innovador, no sólo por constituirse como respuesta a una demanda social no satisfecha sino por utilizar una metodología particular basada en la idea de aprovechamiento terapéutico del fenómeno paradojal, como marco teórico. Cabe aclara que la primera referencia así dicha a esta idea de aprovechamiento terapéutico de la kinesia paradojal se encontró solo recientemente (Duysens, \& Nonnekes, 2021); aunque también se ha hablado de uso de estrategias compensatorias (Nonnekes et al, 2019) y, últimamente, su utilización terapéutica (Tosserams et al, 2021). Previamente, las descripciones del fenómeno se centraban más en lo anecdótico o como recurso de discusión para explicar el comportamiento pero no presentaban directamente esta idea central del uso terapéutico que fue el eje de la iniciativa del TdP desde su conformación en 2002. La única excepción a lo dicho es el conocimiento por comentario personal del trabajo de Prof. David Lee en el Reino Unido (cfr. sección Novedad u originalidad local del conocimiento, más arriba).

\section{Métodos}

Las opciones teórico-metodológicas de la propuesta se fundamentan en los conceptos de interdisciplinariedad y kinesia paradojal. Como metodología, el 
Innovación y Desarrollo Tecnológico y Social (2022) 4: 1-19

pensamiento interdisciplinario es una construcción conjunta de saberes que utiliza conceptos, prácticas y puntos de vista que inicialmente provienen de distintas disciplinas. Es el proceso y resultado de una tarea artesanal de discusión y diálogo, aprendizaje mutuo, humildad y respeto humanos y disciplinarios. Entendemos por interdisciplina una forma de trabajo donde las disciplinas individuales trabajan en conjunto, mirando una misma situación, compartiendo perspectivas y saberes distintos y acordando en la necesidad de ver más de una arista para entender el fenómeno en cuestión.

La conocida revista Nature hizo un llamado a la suma de esfuerzos en la construcción del conocimiento interdisciplinario frente a los problemas contemporáneos en un número que publicó en 2015, reuniendo un conjunto de artículos sobre el tema que demostaban dos condiciones básicas de este trabajo de integración: compromiso y comunicación (Dai, 2020). En las ciencias médicas, la suma de disciplinas no médicas que tradicionalmente no se incluyen dentro de la salud, como las ingenierías y las artes escénicas y visuales, constituye un hito que la pandemia por COVID-19 ayudó a incrementar, no sólo exigiendo cambios de prácticas en los ámbitos clínicos sino también generando desafíos en los espacios pedagógicos (Kneebone, \& Schlegel, 2021).

La segunda opción teórico-metodológica, la kinesia paradojal, constituye un concepto por demás complejo que requiere de una sección especial más abajo. Sin embargo, cabe adelantar que, como fenómeno, la kinesia paradojal significa la ocurrencia de un movimiento que se sale de lo esperado para las condiciones de la persona que lo ejecuta. Sabiendo que esto es posible en ciertos casos y dados determinados estímulos del ambiente, el TdP optó por desarrollar espacios de actividad con propuestas metodológicas basadas en las técnicas artísticas y lúdicas, que, a partir de afectar los estados emocionales de las personas, podían modificar sus comportamientos motores. 
A partir de la incorporación de las ciencias de la computación al proyecto, se incluyó el aprendizaje automático o machine learning como tercera opción teóricometodológica actualmente en proceso de desarrollo. Son diversos los antecedentes que señalan la importancia de la incorporación de las ciencias informáticas en el abordaje de los problemas de las ciencias sociales y naturales (cfr. por ejemplo Leeming, 2021), y, específicamente, en la salud humana y la EP (cfr. por ejemplo Mirelman et al, 2021a, Mirelman et al, 2021b). Por ejemplo, se ha observado que el uso de sensores biométricos asociados al procesamiento informático usando machine learning permite que, a la distancia y en contextos más ecológicos que el consultorio médico, incluyendo o no la sincronización en tiempo real, puedan tomarse parámetros del movimiento de las personas con Parkinson y distinguir rasgos cardinales que los diferencian del movimiento de controles sanos. Esto también puede permitir aumentar las posibilidades de detección en las distintas etapas de la enfermedad y colaborar con el desarrollo de terapéuticas, acompañamiento y seguimiento, evolución y cruzamiento con variables socioambientales.

La Organización Panamericana de la Salud (OPS) (2021) definió ocho principios rectores para la incorporación de la inteligencia artificial en la salud pública, proponiendo que sea una herramienta: (a) centrada en las personas, (b) fundamentada en la ética, (c) transparente, (d) contemplando la protección de datos, (e) manteniendo la integridad científica, (f) abierta y compartible, (g) no discriminatoria y (h) una tecnología controlada por humanos. Como premisa central, la participación de las herramientas de inteligencia artificial en la salud de la población está condicionada a que se maximicen los beneficios para la sociedad sin que se vean comprometidos los derechos de las personas.

El machine learning es un subcampo de la inteligencia artificial que puede colaborar con la salud humana a partir de trabajar con volúmenes de datos (datos de entrenamiento) que se someten a un algoritmo de aprendizaje, identificando 
Innovación y Desarrollo Tecnológico y Social (2022) 4: 1-19

patrones de comportamiento y pudiendo obtener información para clasificar datos nuevos y realizar predicciones (OPS, 2021). Con estos resultados, considerando el nivel de confianza de las predicciones, los condicionantes contextuales y otras variables intervinientes y del comportamiento humano, podrán tomarse decisiones que serán más o menos acertadas según la experticia de los decisores, sus objetivos y demás elementos científicos y humanos.

\section{Desarrollos}

\subsection{Comprensión relacional del comportamiento humano}

Partimos de la concepción de la antropología cognitiva que señala que el comportamiento humano es una relación de co-construcción entre individuo y entorno.

En general, se entiende a la antropología cognitiva como la rama de la antropología que estudia la relación entre la cognición y la cultura. Como disciplina se inicia a mediados del siglo pasado a partir de la revolución cognitiva iniciada por trabajos como los de Howard Gardner (Gardner, 1987). Se ocupa de indagar cómo las personas, en grupos sociales, piensan los objetos y eventos que conforman sus contextos de vida, desde lo cotidiano hasta lo más abstracto, y cómo la cognición emerge de las interacciones en los grupos sociales (Morales Inga, 2019b)

En este contexto, la cultura es como los modelos mentales que las personas forman y usan para percibir, relacionarse e interpretar las cosas, incluyendo no solo los objetos físicos sino fundamentalmente los entornos de comportamiento que incluyen señales, reglas, mensajes, roles e instituciones interrelacionadas en una realidad culturalmente constituida (Morales Inga, 2019a).

La cognición es tratada como un fenómeno cultural y es a través de este entendimiento que se indagan las distintas manifestaciones cognitivas como el juicio moral, el aprendizaje, los sistemas de categorización y otros elementos de la cognición estudiados por la antropología cognitiva y que pueden ser analizados en 
Innovación y Desarrollo Tecnológico y Social (2022) 4: 1-19

estudios transversales y comparativos entre grupos culturales (Morales Inga, 2019a). Entendemos la cognición al modo de Valenti \& Good (1991), no como algo que uno tiene sino como algo que uno hace, poniendo el énfasis en la cognición en contexto que aparece en las prácticas sociales, es decir, proponemos una visión ecológica de la cognición. En este contexto conceptual la cognición, entonces, no está separada del hacer, de la práctica, de la comunicación, donde el cuerpo de los actores tiene un papel preponderante. El cuerpo, como entramado de relaciones, con su movimiento, constituye el instrumento del comportamiento humano.

Partimos de: (a) la naturaleza comunicacional de la conducta (Lahitte, 1981) y (b) el movimiento corporal como instrumento de comunicación (Birdwhistell, 1990), entendiendo que el movimiento influye en las situaciones de intercambio social y su alteración es, probablemente, una situación que afecta el resultado de la comunicación interpersonal. De este modo, la alteración del movimiento corporal por los síntomas de la EP puede contribuir a la ocurrencia de dificultades comunicacionales de las personas con Parkinson. La expresión a través del habla (cuya intensidad y volumen se ven comúnmente afectados), la fluidez del movimiento de extremidades superiores y tronco (las personas suelen presentar un movimiento en bloque en vez de desarticulado y fluido), la expresión facial (se altera la expresión facial, incluso las personas con EP pueden parecer enojadas, abstraídas o aburridas, cuando, en realidad, pueden estar muy interesadas en el interlocutor) y las fluctuaciones (que generan imprevisibilidad y por ende las personas pueden llegar a evitar exponerse a situaciones sociales) afectan sin duda cómo los individuos con EP se manifiestan al entorno y cómo este entorno interpreta esa manifestación. Al interrumpir o disrumpir la comunicación con el medio social, los síntomas parkinsonianos, que también incluyen síntomas no motores como depresión, ansiedad y otros, pueden constituirse en factores de desmejoramiento de la calidad de vida de las personas que conviven con la EP, tanto personas enfermas como familiares y relacionadas. 


\subsection{Comportamiento y enfermedad de Parkinson: kinesia paradojal}

Si bien la EP puede considerarse una condición que en algunos casos llega a ser altamente discapacitante al disrumpir las relaciones entre el individuo y su entorno, posee algunas condiciones comportamentales que pueden permitir aliviar los síntomas y mejorar esas relaciones.

Estamos hablando de la kinesia paradojal, un fenómeno del sistema perceptivomotor que se ve con mayor claridad en enfermedades donde el movimiento corporal está alterado, como en la EP. La kinesia paradojal es un fenómeno que fue inicialmente descripto como entidad nosológica por el neurólogo francés A. A. Souques (1921), quien observó que las personas con EP eran capaces de correr aun cuando no pudieran caminar. Se ha planteado que la kinesia paradojal es un fenómeno del sistema motor (Ballanger et al, 2006) que designa un movimiento inesperado para las condiciones en que se encuentra la persona; incluso, se ha señalado que, pensando al sistema locomotor y sus vías alternativas de un modo más amplio, la kinesia paradójica no sería tan inesperada e inusual (Duysens \& Nonnekes, 2021).

Este fenómeno paradojal involucra una relación entre individuo y entorno donde el sistema perceptivo-motor puede responder a determinadas condiciones ambientales que estimulan el movimiento. Esas condiciones ambientales o propiedades de acción del ambiente se denominan affordances (cfr. Bacigalupe, Tujague, \& Castro, 2017). La ocurrencia de la kinesia paradojal requiere de dos condiciones clave: (a) que la persona sea capaz de moverse y (b) que el ambiente contenga elementos que "inviten" al movimiento. Cabe destacar que las personas con EP pueden moverse; son los mecanismos moduladores del movimiento los que están afectados pero el movimiento per se no está perdido.

Para generar un entorno estimulador que provoque el movimiento en personas con $E P$, en el TdP se vienen proponiendo actividades grupales lúdicas y artísticas, 
Innovación y Desarrollo Tecnológico y Social (2022) 4: 1-19

haciendo uso de las artes escénicas, plásticas y corporales y la educación física y juegos deportivos. Si bien la actividad grupal se sabe intuitivamente que promueve la motivación, actualmente existen estudios controlados que muestran que la rehabilitación de personas con EP basada en grupo (versus individual) puede promover la motivación y mejorar la movilidad de las personas (Mitsui et al, 2021). Asimismo, se sabe que el afecto positivo se relaciona con el soporte social, así como que la generación de una experiencia estética puede representar un factor terapéutico que mejore el bienestar y la autoeficacia corporal (Vescovelli, Sarti \& Ruini, 2018).

Para el desarrollo de estas actividades, la vía necesaria ha sido el trabajo interdisciplinario, aprovechando las fortalezas de las distintas disciplinas de la salud, el arte y la educación y favoreciendo la construcción de un lenguaje en común que permitiera el trabajo constructivo en función de la salud de las personas con EP. En este contexto de interdisciplinariedad se inscribe la formación de estudiantes de la práctica final obligatoria de Medicina de la Universidad Nacional de La Plata (UNLP), alumnos de otras carreras y voluntarios graduados y no graduados de la misma u otra institución educativa.

\subsection{Cambios, crecimientos e inclusiones: pandemia y ciencias de la computación}

Con el advenimiento de la pandemia por COVID-19 y el aislamiento social preventivo y obligatorio (ASPO), el territorio del grupo de soporte se virtualizó y diversificó en distintos canales de comunicación que permitieron mantener los vínculos existentes, fortalecerlos y construir nuevos lazos.

Ese crecimiento se vio reflejado no solamente en el grupo de personas con EP y familiares, sino también en el trabajo intedisciplinario, donde a la innovación tecnológico-social ya establecida se sumaron las tecnologías de la información y la comunicación (en forma de entornos virtuales, espacios de videoconferencias, 
Innovación y Desarrollo Tecnológico y Social (2022) 4: 1-19

redes sociales, WhatsApp y otros medios de comunicación, además de la tradicional llamada telefónica, cfr. Bacigalupe et al, 2021) y las ciencias de la computación, específicamente la ingeniería en sistemas con sus herramientas de administración, organización, sistematización, distribución y comunicación de la información pero sobre todo con el potencial de sus instrumentos más avanzados como el aprendizaje automático o machine learning.

Para esto se incorporó un grupo de trabajo de la Universidad Tecnológica Nacional Regional La Plata (UTN-RLP, Grupo de investigación y desarrollo aplicado a sistemas informáticos y computacionales [GIDAS]) con el que se creó el proyecto TelePark. Esta iniciativa tuvo un doble propósito: (a) desarrollar una herramienta de software que permitiera administrar la actividad del TdP, organizar y procesar datos y generar nueva información en forma de alertas tempranas para el acompañamiento de las actividades del Taller y (b) generar un espacio de formación de estudiantes de ingeniería en sistemas para el cumplimiento de sus prácticas finales de carrera (ya que en pandemia disminuyeron las posibilidades de prácticas extramuros por escasa oferta vinculada al ASPO), becas de investigación y desarrollos de posgrado. Al momento se ha desarrollado la primera etapa del trabajo en tecnología de sistemas, con el modelado de diversos diagramas del sistema y la construcción de una versión preliminar del software, proceso que continúa en revisión y ampliación a medida que se van abordando las etapas de la gestión de requerimientos.

Se está trabajando en el backend, que es la parte del sistema que se conecta con la base de datos y el servidor y contiene la lógica de la aplicación que administra esos datos (Chapaval, 2017), donde algunos de los módulos y operaciones ya están funcionando. Este desarrollo permite la consulta, modificación y eliminación de distintas entidades, persistencia en la base de datos, autenticación y autorización ante las operaciones solicitadas a la interfaz de programación de aplicaciones (application programming interface [API]). 
Innovación y Desarrollo Tecnológico y Social (2022) 4: 1-19

Se ha probado, además, la integración con una versión del frontend, que es la parte del sistema que interactúa con los usuarios (Chapaval, 2017) que, en este caso, serán los profesionales del grupo de soporte físico y social. Se han podido realizar operaciones de prueba de manera exitosa, utilizando el backend para obtener distintos datos necesarios para el formulario y permitiendo el guardado en la base de datos de la entidad resultante.

Se gestionaron las cuentas en GitHub (https://github.com/, que es una plataforma de desarrollo de software para alojar el proyecto), donde el código ya se encuentra disponible para los miembros del proyecto, ya sea para su consulta, uso, o modificación. Estos avances se describen con mayor detalle en Mamani Villca et al (2021).

\section{Perspectivas presentes y futuras}

Al momento estamos desarrollando el grupo de soporte de forma virtual con actividades de educación física, juegos corporales y movimiento con música. Se han incorporado personas que durante la pandemia no podían asistir a sus lugares de rehabilitación por la declaración del ASPO, además de otros participantes que conocieron del espacio y se sumaron a la propuesta virtual. Asimismo, desarrollamos un grupo de WhatsApp donde se comparten vivencias cotidianas, tanto de familiares como de personas con Parkinson y equipo profesional. Abundan los juegos de ingenio propuestos por los mismos participantes y resueltos en conjunto por los otros miembros del grupo. Estas acciones constituyen un espacio de soporte virtual sin precedente en nuestro grupo y que ha significado una contención y ayuda mutua en momentos desafiantes como los vividos en las peores épocas de pandemia, y que se co-construye permanentemente como espacio de intercambio diario.

Hemos mantenido el contacto telefónico y por mensaje de textos con aquellos integrantes del grupo que por una u otra razón no siguieron comunicados por 
Innovación y Desarrollo Tecnológico y Social (2022) 4: 1-19

WhatsApp y en el grupo de actividad virtual. Asimismo, realizamos visitas domiciliarias a partir de que fue posible hacerlo y las familias de los participantes lo aceptaron.

El desafío que actualmente se presenta es la vuelta a la presencialidad, en un contexto donde los cuidados deben extremarse para garantizar la salubridad de los espacios y donde algunos de los participantes se integraron al taller cuando ya se desarrollaba de forma virtual. Ante estas cuestiones nos proponemos el desarrollo de una alternativa híbrida o mixta que permita a quienes no puedan acercarse o no lo deseen, que puedan continuar con la actividad del grupo de forma remota. De esta forma, seguimos ampliando la innovación al proponer vías nuevas de comunicarnos con la población y ofrecer soporte físico y social incluso a personas alejadas del espacio geográfico de desarrollo del TdP.

Los intercambios tecnológicos que comenzamos en 2020 con el grupo de expertos en sistemas informáticos continuarán a fin de contar con una herramienta básica que podamos empezar a probar in situ, para poder retroalimentar el desarrollo y mejorarlo de acuerdo con las necesidades y funcionalidades de la práctica concreta.

\section{Información sobre el patentamiento y registro de la innovación}

Si bien no hay patentamiento de la innovación al momento, existen numerosas publicaciones en distintos medios académicos que dan cuenta de la idea original y sus desarrollos. Ejemplo de estas son Dillon et al (2010), Pujol, Dillon \& Bacigalupe (2012), Dubois \& Bacigalupe (2014), Nomdedeu et al (2018), Bacigalupe (2019), Maggi et al (2017) y Bacigalupe et al (2020).

\section{Financiamiento}

La Universidad Nacional de La Plata ha apoyado la innovación a través de su área de extensión universitaria, acreditando y subsidiando variados proyectos. El proyecto que al momento está en curso es Taller de Parkinson. Fortalecimiento de 
Innovación y Desarrollo Tecnológico y Social (2022) 4: 1-19

lazos en territorio virtual (acreditado y subsidiado por UNLP, agosto-diciembre 2021, Expte. Código 100 n 6824, 15/7/21).

Cabe aclarar que La Universidad Tecnológica Nacional apoya a algunos de los estudiantes que están colaborando con el desarrollo tecnológico con el otorgamiento de becas.

\section{Agradecimientos}

Reconocemos el magisterio permanente del Dr. José Luis Dillon y su espíritu innovador y solidario, a quien corresponde la idea original del Taller de Parkinson. Asimismo, agradecemos al Prof. de Educación Física Enrique Maggi, que se desempeña voluntariamente en el TdP desde hace quince años, y a todos los participantes con Parkinson, familiares y voluntarios presentes y pasados. Cabe reconocer la colaboración de los estudiantes avanzados de UTN-RLP que están desarrollando la herramienta informática, Sonia Mamani Villca, Jonathan Rodríguez, Elías Brizuela, Franco Raggio, Carolina Méndez, David Huilcañahui y Lautaro Scarselletta, y a la dirección y el personal del GIDAS. Finalmente agradecemos el apoyo constante de la Facultad de Ciencias Médicas de la UNLP. MAB pertenece a la Carrera de Investigador Científico y Tecnológico de CONICET y agradece su apoyo.

\section{Referencias bibliográficas}

Bacigalupe, M. A. (2019). Vivir con Parkinson y convivir para contarlo: entrevista a Carolina Cassouss. Dios y el Hombre, 3(2), 1-13. https://doi.org/10.24215/26182858e049

Bacigalupe, M. A., Pujol, S. \& Maggi, E. (2021). Espacio de comunicación, cuerpo, aprendizaje y convivencia con la Enfermedad de Parkinson (EP). XIV Jornadas de Sociología 'Sur, pandemia y después'. Facultad de Ciencias Sociales, Universidad de Buenos Aires, 1 5/11/21. Disertación. Eje 6 - Mesa 180: Construcciones sociales de la (dis)capacidad: ¿viejas y nuevas demandas? http://jornadasdesociologia2021.sociales.uba.ar/mesas/

Bacigalupe, M. A., Pujol, S., Maggi, E., \& Dillon, J. L. (2020). Enfoque relacional de la Enfermedad de Parkinson como oportunidad para la integración de competencias pedagógicas, científicas y sociales. En CJ Giordano y G Morandi (Comp). Memorias de 
las $3^{\circ}$ Jornadas sobre las Prácticas Docentes en la Universidad Pública "El proyecto político académico de la Educación Superior en el contexto nacional y regional" (pp. 1580-1589). La Plata: UNLP. http://sedici.unlp.edu.ar/handle/10915/111431

Bacigalupe, M. A., Tujague, M. P., \& Castro L. (2017). Las cualidades de los objetos influyen en el tiempo de reacción de las personas con Enfermedad de Parkinson. Interdisciplinaria, 34(2), 473-488. https://doi.org/10.16888/interd.2017.34.2.14

Bauso, D. J., Tartari, J. P., Stefani, C. V., Rojas, J. I., Giunta, D. H., \& Cristiano, E. (2012). Incidence and prevalence of Parkinson's disease in Buenos Aires City, Argentina. European Journal of Neurology, 19(8), 1108-1113. https://doi.org/10.1111/j.14681331.2012.03683.x

Birdwhistell, R. (1990). Kinesics and Context: Essays on Body Motion Communication (5a reimp). USA: University of Pennsylvania Press.

Chapaval, N. [maldeadora]. (2017, s/d). Qué es Frontend y Backend: diferencias y características. Platzi blog. https://platzi.com/blog/que-es-frontend-y-backend/

Dai, L. (2020). What are fake interdisciplinary collaborations and why do they occur? Nature index. https://www.natureindex.com/news-blog/what-are-fake-interdisciplinarycollaborations-and-why-do-they-occur

Dillon, J. L., Bacigalupe, M. A., Pujol, S., Moore, M. E., \& Mazza, A. (en representación del Equipo de Trabajo del Taller de Parkinson). (2010). Metodología desarrollada para mejorar la calidad de vida de las personas con Enfermedad de Parkinson (EP) mediante la aplicación de los principios teóricos del fenómeno paradojal en el Taller de Parkinson (TdP). Revista Científica de la Facultad de Ciencias Médicas, Tercera Época, 2(2), 1 .

http://www.med.unlp.edu.ar/revista/archivos/201012/dillon \%28psiquiatria\%29.pdf

Disposición 1838/2007 del Servicio Nacional de Rehabilitación de Argentina. (2007, 18 de octubre). Apruébase la Normativa para Certificación de Discapacidad en Pacientes con Enfermedad de Parkinson. https://www.argentina.gob.ar/normativa/nacional/disposici\%C3\%B3n-1838-2007135578/texto

Dubois, V., Bacigalupe, M. A. (2014). El espacio de teatro en el Taller de Parkinson. Revista $+E$, 4(4), 106-111. http://www.unl.edu.ar/categories/view/no4\#.VK6JoSvF98E

Duysens, J., \& Nonnekes, J. (2021). Parkinson's Kinesia Paradoxa Is Not a Paradox. Movement Disorders, 36(5), 1115-1118. https://doi.org/10.1002/mds.28550

Gardner, H. (1987). The mind's new science. NY: Basic Books Inc. Publisher.

Keefe, K., Salamone, J., Zigmond, M., \& Stricker, E. (1989). Paradoxical kinesia in parkinsonism is not caused by dopamine release. Studies in an animal model. Archives of Neurology, 46(10), 1070-1075. https://doi.org/10.1001/archneur.1989.00520460046012

Kneebone, R., \& Schlegel, C. (2021). Thinking across disciplinary boundaries in a time of crisis. The Lancet, 397(10269), 89-90. https://doi.org/10.1016/s0140-6736(20)32757-4

Lahitte, H. B. (1981). Aportes teórico-metodológicos al estudio del comportamiento. Symposia: Sextas Jornadas Argentinas de Zoología, separata. Buenos Aires: Ramos Americana.

Leeming, J. (2021). How Al is helping the natural sciences. Nature, 598(7880), S5-S7. https://doi.org/10.1038/d41586-021-02762-6

Maggi, E., Bacigalupe, M. A., Grosso, M., Veneziano, M., \& Pujol, S. (2017). Enfermedad de Parkinson y claves externas: ¿facilitación o no de la marcha? Actas del $12^{\circ}$ Congreso 
argentino y $7^{\circ}$ latinoamericano de Educación Física y Ciencias. Departamento de Educación Física, Facultad de Humanidades y Ciencias de la educación, UNLP. http://congresoeducacionfisica.fahce.unlp.edu.ar/120-congreso/actas2017/Mesa\%2007 Maggi.pdf/view

Mamani Villca, S., Brizuela, E., Rodríguez, J., \& Raggio, F. (2021). Prácticas supervisadas en el desarrollo de un software de seguimiento de la salud de personas con Enfermedad de Parkinson. $9^{\circ}$ Congreso Nacional de Ingeniería Informática / Sistemas de Información (CoNalISI), 4-5/11/21, Facultad Regional Mendoza de la UTN. Presentado en formato de poster. En prensa trabajo completo. https://www4.frm.utn.edu.ar/conaiisi/

Mirelman, A., Dorsey, E. R., Brundin, P., \& Bloem, B. (2021a). Using Technology to Reshape Clinical Care and Research in Parkinson's Disease. Journal of Parkinson's Disease, 11(s1), S1-S3. https://doi.org/10.3233/jpd-219002

Mirelman, A., Frank, M., Melamed, M., Granovsky, L., Nieuwboer, A., Rochester, L., Din, S., Avanzino, L., Pelosin, E., Bloem, B., Della Croce, U., Cereatti, A., Bonato, P., Camicioli, R., Ellis, T., Hamilton, J., Hass, C., Almeida, Q., Inbal, M., Thaler, A., Shirvan, J., Cedarbaum, J., Giladi, N., Hausdorff, J. (2021b). Detecting Sensitive Mobility Features for Parkinson's Disease Stages Via Machine Learning. Movement Disorders, 36(9), 2144-2155. https://doi.org/10.1002/mds.28631

Mitsui, T., Arii, Y., Tsukamoto, A., Taniguchi, K., Mabuchi, M., Shimizu, A., Sumitomo, N., \& Maki, Y. K. (2021). Sociability- based fitness approach in Parkinson's disease: Comparison with conventional rehabilitation. European Journal of Neurology, 28(6), 1893-1900. http://dx.doi.org/10.1111/ene.14798

Morales Inga, S. (2019a). La antropología, ¿una ciencia cognitiva? Ciencia Cognitiva, 13(1), 810. http://www.cienciacognitiva.org/?p=1782

Morales Inga, S. (2019b). La antropología como ciencia cognitiva: una breve introducción. Scientia in Verba Mag, 4, 106-117. https://vdoc.pub/documents/la-antropologiacomo-ciencia-cognitiva-una-breve-introduccion-1vaj4u42dkg8

$\begin{array}{llll}\text { Nature. (2015). Interdisciplinarity. } & \text { Nature, }\end{array}$ https://www.nature.com/collections/jcfdbccgjj

Nomdedeu, G., Bacigalupe, M. A., Piciucchi, M. V., \& Sansalone, N. (2018). Despertando al cuerpo desde el movimiento: expresión corporal y Parkinson. Revista e+e, 5(5), 120 132. https://revistas.unc.edu.ar/index.php/EEH/article/view/19798/19543

Nonnekes, J., Rǔžička, E., Nieuwboer, A., Hallett, M., Fasano, A., \& Bloem, B. R. (2019). Compensation Strategies for Gait Impairments in Parkinson Disease. A Review. JAMA Neurology, 76(6), 718-725. https://doi.org/10.1001/jamaneurol.2019.0033

Organización Panamericana de la salud [OPS]. (2021). La inteligencia artificial en la salud pública. https://iris.paho.org/handle/10665.2/53887

Perception Movement Action Research Consortium. (s/d). Rehabilitation and Training of Actions Videos. http://www.pmarc.ed.ac.uk/video/rehabilitation-and-training-ofactions/index.html [accedido por última vez 2021, 29 de octubre]

Pujol, S., Bacigalupe, M. A., \& Dillon, J. L. (2012). Programa Salud, creatividad y movimiento: el Taller de Parkinson. En Programas de Extensión (pp 64-73). Universidad Nacional de La Plata. http://unlp.edu.ar/uploads/docs/libro de programas de extension.pdf

Resolución 753/2007 del Ministerio de Salud de Argentina. (2007, 27 de junio). Apruébase la creación del Programa Nacional de Envejecimiento Activo y Salud para los Adultos 
Innovación y Desarrollo Tecnológico y Social (2022) 4: 1-19

Mayores.

Objetivos.

http://servicios.infoleg.gob.ar/infoleglnternet/resaltaranexos/125000-

129999/129728/norma.htm

Tosserams, A., Wit, Sturkenboom, I. H. W. M., Nijkrake, M. J., Bloem, B. R., \& Nonnekes, J. (2021). Perception and Use of Compensation Strategies for Gait Impairment by Persons With Parkinson Disease. Neurology. https://doi.org/10.1212/WNL.0000000000012633

Tysnes, O., \& Storstein, A. (2017). Epidemiology of Parkinson's disease. Journal of Neural Transmission, 124(8), 901-905. https://doi.org/10.1007/s00702-017-1686-y

Valenti, S. S., \& Good, J. M. (1991). Social affordances and interaction I: Introduction. Ecological Psychology, 3(2), 77-98. https://doi.org/10.1207/s15326969eco0302 2

Vescovelli, F., Sarti, D., \& Ruini, C. (2018). Subjective and psychological well-being in Parkinson's Disease: A systematic review. Acta Neurologica Scandinavica, 138(1), 1223. https://doi.org/10.1111/ane.12946

Vial, F., Delgado, I. Idiaques, J. F., Canals, F., \& Chana-Cuevas, P. (2021). Epidemiology of Parkinson's Disease in Chile [Abstract]. Neuroepidemiology, 55(5), 393-397. https://doi.org/10.1159/000517750

\section{Selección de entrevistas en medios de comunicación masiva:}

Convivir con Parkinson. Entrevista por la presentación del libro El inglés de mi vida: Convivir con Parkinson. Infobae.

25/05/19. https://www.infobae.com/sociedad/2019/05/25/convivir-con-parkinson-una-mujerde-73-anos-cuenta-como-evitar-la-depresion-de-la-enfermedad-y-luchar-contra-losestigmas/

Entrevista grupal. Carolina Cassouss, Silvana Pujol y M. Angeles Bacigalupe. Radio Almuerzos. Agosto 2019. https://www.cpba.com.ar/delegaciones/la-plata/item/9135-radioalmuerzos-agosto-2019

Entrevista individual por el Día Mundial del Parkinson. Columna sobre integración de Fernando Bossi. Radio Altavoz. La Plata, 13/04/2017. https://www.mixcloud.com/eduardocortes9/noticias-que-despiertan-columna-de-inclusion-de-fernando-bossi/

Entrevista individual. Parkinson y salud: los desafíos del movimiento. Nota a cargo de Nicolás Camargo Lescano. Agencia CTyS-UNLaM. 7/06/18. http://www.ctys.com.ar/index.php?idPage=20\&idArticulo=3541\&seccion=13.

Una reserva cognitiva para afrontar el paso de los años. Entrevista. Diario El Día. Sección Información General. Entrevistador: Nicolás Maldonado. La Plata. 22/7/12. http://www.eldia.com.ar/edis/20120722/informaciongeneral10.htm 